\title{
Quantum confinement effect on the effective mass in two-dimensional electron gas of AIGaN/GaN heterostructures
}

A. M. Kurakin, S. A. Vitusevich, S. V. Danylyuk, H. Hardtdegen, N. Klein, Z. Bougrioua, A. V. Naumov, and A. E. Belyaev

Citation: Journal of Applied Physics 105, 073703 (2009);

View online: https://doi.org/10.1063/1.3100206

View Table of Contents: http://aip.scitation.org/toc/jap/105/7

Published by the American Institute of Physics

\section{Articles you may be interested in}

Temperature dependent effective mass in AIGaN/GaN high electron mobility transistor structures Applied Physics Letters 101, 192102 (2012); 10.1063/1.4765351

Effective mass of the two-dimensional electron gas and band nonparabolicity in $\mathrm{Al}_{X} \mathrm{Ga}_{1-X} N / \mathrm{GaN}$ heterostructures

Applied Physics Letters 88, 172115 (2006); 10.1063/1.2197306

Band parameters for III-V compound semiconductors and their alloys

Journal of Applied Physics 89, 5815 (2001); 10.1063/1.1368156

Energy dependence of electron effective mass and effect of wave function confinement in a nanoscale In $0.53 \mathrm{Ga} 0.47 \mathrm{As} / \mathrm{In}_{0.52} \mathrm{Al}_{0.48} \mathrm{As}$ quantum well

Journal of Applied Physics 113, 234314 (2013); 10.1063/1.4811717

Two dimensional electron gases induced by spontaneous and piezoelectric polarization in undoped and doped AlGaN/GaN heterostructures

Journal of Applied Physics 87, 334 (2000); 10.1063/1.371866

Band parameters for nitrogen-containing semiconductors

Journal of Applied Physics 94, 3675 (2003); 10.1063/1.1600519

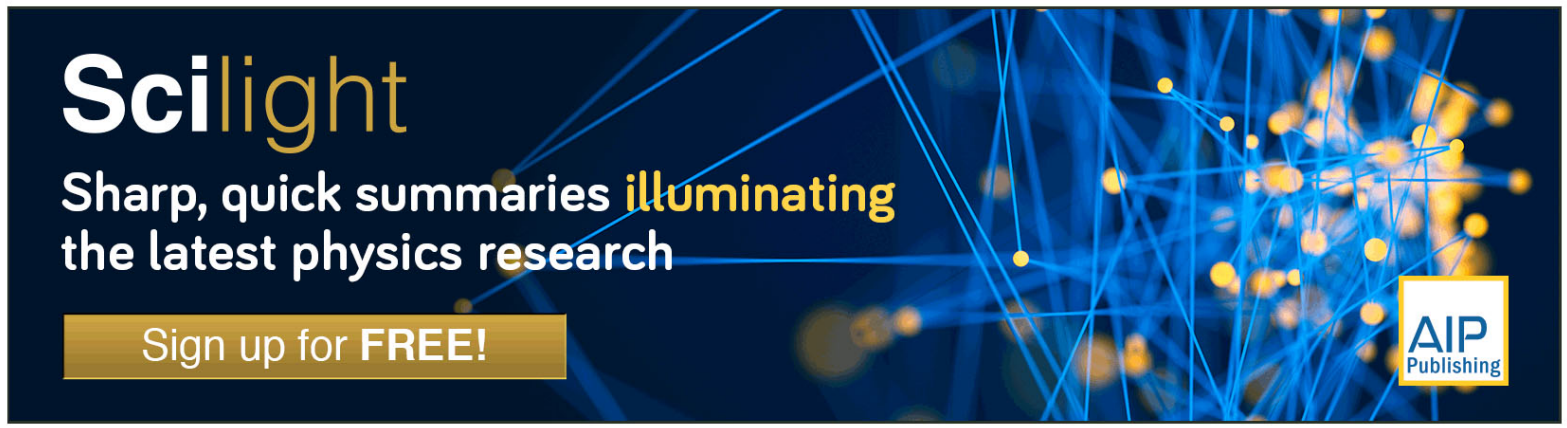




\title{
Quantum confinement effect on the effective mass in two-dimensional electron gas of AIGaN/GaN heterostructures
}

\author{
A. M. Kurakin, ${ }^{1}$ S. A. Vitusevich, ${ }^{1, a)}$ S. V. Danylyuk, ${ }^{1}{ }^{H}$. Hardtdegen, ${ }^{1}$ N. Klein, ${ }^{1}$ \\ Z. Bougrioua, ${ }^{2}$ A. V. Naumov, ${ }^{3}$ and A. E. Belyaev ${ }^{3}$ \\ ${ }_{1}^{1}$ Institut für Bio- und Nanosysteme and Center of Nanoelectronic Systems for Information Technology \\ (CNI), Forschungszentrum Jülich, Jülich D-52425, Germany \\ ${ }^{2}$ Centre de Recherche sur l'Hétéroépitaxie et ses Applications, CNRS, rue Bernard Gregory, \\ F-06560 Valbonne, France \\ ${ }^{3}$ Institute of Semiconductor Physics, NASU, Pr. Nauki 45, 03028 Kiev, Ukraine
}

(Received 5 January 2009; accepted 16 February 2009; published online 1 April 2009)

\begin{abstract}
We report the results of direct measurements and a theoretical investigation of the in-plane effective mass in the two-dimensional electron gas of nominally undoped AlGaN/GaN heterostructures with a different degree of quantum confinement. It is shown that in most cases the conduction band nonparabolicity effect is overestimated and the electron wave-function penetration into the barrier layer should be taken into account. The contribution of the wave-function hybridization is determined to play the dominant role. The band edge effective mass value is deduced to be $(0.2 \pm 0.01) m_{0}$. C 2009 American Institute of Physics. [DOI: 10.1063/1.3100206]
\end{abstract}

\section{INTRODUCTION}

During the past decade practically every paper dedicated to the study of nitride-based semiconductors begins by emphasizing their importance for modern electronics and optoelectronics. Indeed, prominent physical properties of these materials such as wide bandgap energy, high electron mobility, high saturation drift velocity, high breakdown voltage, and good thermal conductivity open good perspectives in their usage for numerous high-temperature, high-frequency, and high-power applications. To this end, the knowledge of fundamental parameters determining the electronic transport in III-nitrides is of great importance. ${ }^{1}$ One of such parameters is the effective electron mass that is the fundamental value in the description of semiconductor properties.

The first report on the electron effective mass measurement in GaN epitaxial films appeared in 1973 (Ref. 2) and a value of $m^{*}=0.2 \pm 0.02 m_{0}\left(m_{0}\right.$ is a free electron mass) was confirmed by theoretical calculations ${ }^{3}$ and by a series of experiments performed by different methods on bulk ${ }^{4}$ and epitaxial $^{5-8}$ wurtzite GaN. The largest value of $0.236 m_{0}$ was obtained from the measurement of the transition energy of shallow donors by the authors of Ref. 9. The smallest value of $0.15 m_{0}$ was obtained by empirical pseudopotential bandstructure calculations. ${ }^{10}$ Apart from that, the value of $0.20 m_{0}$ is recommended in the review ${ }^{11}$ and entered in most textbooks ${ }^{12}$ as a common-used parameter for the effective mass of electrons in "bulklike" GaN films.

In the case of a two-dimensional electron gas (2DEG) formed at the interface of a $\mathrm{AlGaN} / \mathrm{GaN}$ heterostructure or in AlGaN/GaN/AlGaN quantum wells the situation dramatically changes. Even in high-quality AlGaN/GaN heterostructures, the in-plane effective mass of 2D electrons measured by various direct and indirect techniques has shown effective

\footnotetext{
${ }^{a)}$ Electronic mail: s.vitusevich@fz-juelich.de. On leave from Institute of Semiconductor Physics, NASU, 03028 Kiev, Ukraine.
}

mass enhancement and a large scatter of the data ${ }^{13-30}$ (Fig. 1). In general, the observed scatter of the $2 \mathrm{DEG}$ effective mass in $\mathrm{AlGaN} / \mathrm{GaN}$ structures is not surprising because it reflects the variation in the band structure caused by heterostructure design and intrinsic effects of piezoelectric and spontaneous polarization at the interface, remote impurities in the barrier, residual impurities in the well, alloy disorder, interface roughness, etc. Obviously, these parameters can be quite different for various $\mathrm{AlGaN} / \mathrm{GaN}$ heterostructures grown by different methods using different growth conditions. Nevertheless, the observed enhancement of the effective mass of 2D electrons (the summary of available data published in the literature are shown in Fig. 1 by closed circles) is beyond the question and the mechanism responsible for the effect should be found.

The effective mass enhancement can be understood if the effective mass used for the description of the 2DEG properties is approximated by the corresponding value used for the bulk material. However, this assumption becomes less valid with increasing Fermi energy for increased 2DEG density at the $\mathrm{AlGaN} / \mathrm{GaN}$ interface. It is known (from cyclotron

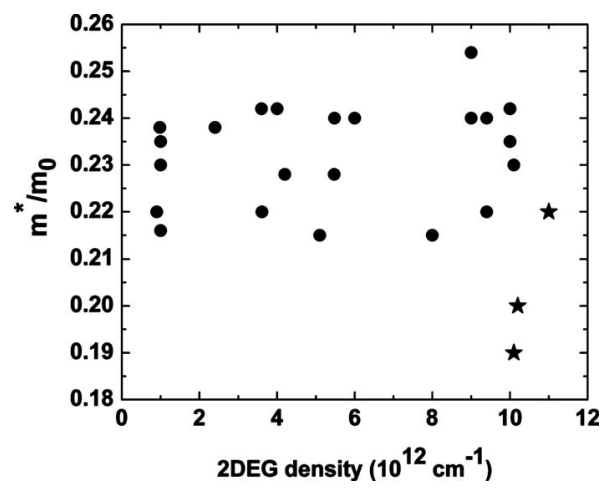

FIG. 1. Experimental values of effective electron mass in AlGaN/GaN as a function of the 2DEG sheet density. The closed circles are data from Refs. 13-29, and the stars correspond to our data. 
resonance $^{14-16}$ and magnetotransport ${ }^{17-19}$ results) that the inplane effective mass can exceed the bulk values considerably due to the increasing effect of conduction band nonparabolicity at higher Fermi energy. At the same time, even taking into account this effect, a large scatter of the data is not well understood and uncertainty of the effective mass in 2DEG with different carrier density and spatial confinement is large. ${ }^{30}$ It has been pointed out that this discrepancy can be attributed to the effect of the wave-function penetration into the barrier layer (i.e., wave-function hybridization). However, there was no clear demonstration of the penetration effect for $\mathrm{AlGaN} / \mathrm{GaN}$ heterostructures.

In this work, we report the results of direct measurements and a theoretical investigation of the in-plane effective mass of the 2DEG in nominally undoped $\mathrm{AlGaN} / \mathrm{GaN}$ heterostructures with a different degree of quantum confinement aimed to show the contribution of the wave-function hybridization into effective mass enhancement.

\section{EXPERIMENTAL DETAILS}

Samples under study were fabricated from high-quality structures grown by metal organic chemical vapor deposition on (0001) $c$-plane sapphire substrates. Three types of AlGaN/ GaN heterostructures designed for high electron mobility transistor (HEMT) application were investigated. The structures have the following sequence of layers:

- sample A: undoped GaN buffer, $1.1 \mu \mathrm{m}$; undoped $\mathrm{Al}_{0.33} \mathrm{Ga}_{0.67} \mathrm{~N}$ barrier, $23 \mathrm{~nm} ; \mathrm{Si}_{3} \mathrm{~N}_{4}$ passivation layer, $320 \mathrm{~nm}$;

- sample B: undoped GaN buffer, $3.0 \mu \mathrm{m}$; undoped $\mathrm{Al}_{0.30} \mathrm{Ga}_{0.70} \mathrm{~N}$ barrier, $30 \mathrm{~nm}$; GaN cap layer, $4 \mathrm{~nm}$;

- sample C: GaN nucleation layer, $28 \mathrm{~nm} ; \mathrm{GaN}$ insulating buffer, $7.5 \mu \mathrm{m}$; undoped AlN interlayer, $\sim 1 \mathrm{~nm}$; undoped $\mathrm{Al}_{0.25} \mathrm{Ga}_{0.75} \mathrm{~N}$ barrier, $25 \mathrm{~nm}$; GaN cap layer, $1.3 \mathrm{~nm}$.

In the unintentionally doped structures under study, the concentration of uncontrollable background impurities was $<1$ $\times 10^{17} \mathrm{~cm}^{-3}$. The Hall bar mesas $\left(140 \times 700 \mu \mathrm{m}^{2}\right)$ were fabricated with Ohmic contacts processed using Ti/Al/Ni/Au metallization. The dielectric thin film SiN coating or $\mathrm{GaN}$ capping was deposited for surface passivation (for details, see Refs. 31-33)

$I-V$ and $C-V$ characteristics of samples at room temperatures were measured using the Keithley 4200 parameter analyzer and the HP4284 LRC meter. Magnetotransport characteristics were studied in the range of temperatures $T$ $=0.3-300 \mathrm{~K}$ and magnetic fields $B=0-10 \mathrm{~T}$ using a standard lock-in technique. The samples were measured at a low frequency $(\sim 17 \mathrm{~Hz})$ to minimize the out-of-phase signal and at a low current $(\sim 100 \mathrm{nA})$ modulation to ensure the linear response and to prevent heating effects.

\section{RESULTS AND DISCUSSION}

Figure 2 shows the evolution of the longitudinal magnetoresistance of the $2 \mathrm{DEG}$ in the $\mathrm{AlGaN} / \mathrm{GaN}$ heterostructure (sample B) measured in a perpendicular magnetic field up to $10 \mathrm{~T}$ at $0.35 \mathrm{~K}$. At low field a decrease in magnetoresistance

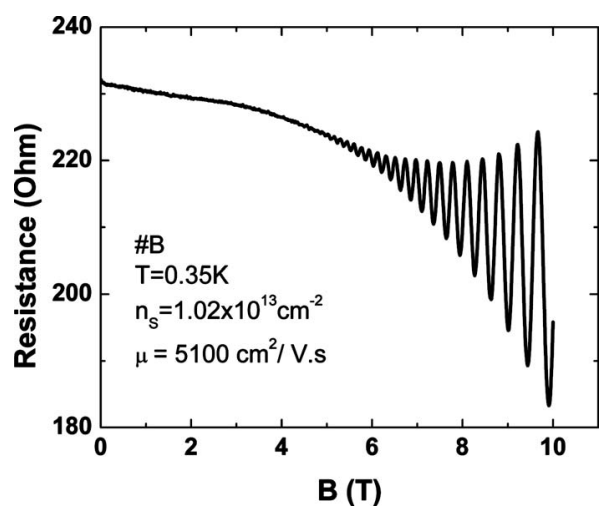

FIG. 2. Magnetoresistance of the 2DEG in AlGaN/GaN (sample B) at $T$ $=0.35 \mathrm{~K}$

with increasing magnetic field is registered. With increasing magnetic field the periodic Shubnikov-de Haas (SdH) oscillations in magnetoresistance are clearly resolved starting with magnetic field of $5 \mathrm{~T}$. The amplitude of the $\mathrm{SdH}$ oscillations decreased with increasing temperature. Similar behavior of the magnetoresistance was recorded for samples B and $\mathrm{C}$.

The sheet concentration of the 2DEG was determined using the $\mathrm{SdH}$ oscillation period. From the temperature dependence of the amplitude of the $\mathrm{SdH}$ oscillations, the effective mass was calculated. The quantum lifetime was deduced from the $\mathrm{SdH}$ data using the oscillatory part of the magnetoresistance, and the transport lifetime was derived from the zero-field Hall mobility measurements.

The determined characteristics of the 2DEG in the $\mathrm{AlGaN} / \mathrm{GaN}$ heterostructures are summarized in Table I, where $E_{F}$ is the Fermi energy measured starting from the quantization energy $E_{0}, n_{S}$ is the sheet carrier density of 2DEG, $m_{E}{ }^{*}$ is the effective electron mass at the Fermi energy, $\tau_{\text {tr }}$ is the transport lifetime, $\tau_{q}$ is the quantum lifetime, and $\mu$ is the carrier mobility measured at temperatures 0.35 and $300 \mathrm{~K}$.

It should be noted that the high electron density of the 2DEG, $n_{S} \cong 1.1 \times 10^{13} \mathrm{~cm}^{-2}$, is obtained in AlGaN/GaN heterostructures without modulation doping, due to the strong spontaneous and piezoelectric polarization at the $\mathrm{AlGaN} /$ $\mathrm{GaN}$ heterointerface. The carrier concentration measured at low (Hall effect) and high (SdH oscillations) magnetic fields was the same order of magnitude for the investigated samples. Additionally there was no registered beating in the plot of magnetoresistance. These facts indicate that 2D electrons occupy only the lowest subband in the quantum well.

The increase in electron mobility with decreasing tem-

TABLE I. The determined characteristics of the 2DEG in the AlGaN/GaN heterostructures under study.

\begin{tabular}{lccccc}
\hline \hline & & & & & \\
Sample & $\begin{array}{c}E_{F} \\
(\mathrm{meV})\end{array}$ & $\begin{array}{c}n_{s}\left(10^{13}\right) \\
\left(\mathrm{cm}^{-2}\right)\end{array}$ & $\begin{array}{c}m_{E}{ }^{*} \\
\left(m_{0}\right)\end{array}$ & $\tau_{\mathrm{tr}} / \tau_{q}$ & $\begin{array}{c}\mu \\
(0.35 \mathrm{~K} / 300 \mathrm{~K}) \\
\left(\mathrm{cm}^{2} / \mathrm{V} \mathrm{s}\right)\end{array}$ \\
\hline $\mathrm{A}$ & 130 & 1.1 & $0.22 \pm 0.01$ & 4.3 & $2300 / 1380$ \\
$\mathrm{~B}$ & 131 & 1.02 & $0.20 \pm 0.01$ & 7.7 & $5100 / 1260$ \\
$\mathrm{C}$ & 129 & 1.01 & $0.19 \pm 0.01$ & 13.7 & $11500 / 1850$ \\
\hline \hline
\end{tabular}


perature from 300 to $0.35 \mathrm{~K}$ (1.7 times in sample $\mathrm{A}$, 4 times in sample B, and 6 times in sample C) demonstrates the reduced scattering of $2 \mathrm{DEG}$ carriers. Besides, the increasing ratio between the transport and quantum lifetimes, $\tau_{\mathrm{tr}} / \tau_{q}$, from 4.3 in sample A to 13.7 in sample $\mathrm{C}$, provides information about the dominant mechanism of short- and long-range scattering of 2D electrons at the heterointerface.

Actually the data prove that a high-quality 2 DEG channel is formed at the interface in each structure. However, the obtained values of the effective mass listed in Table I reveal the inconsistency between the considerable increase in the effective mass from $0.19 m_{0}$ in sample $\mathrm{C}$ to $0.22 m_{0}$ in sample $\mathrm{A}$ and the minor changes in the 2DEG density. This fact cannot be explained only by the mentioned effect of nonparabolicity of the electron conduction band. To find the origin of the effective mass enhancement observed in our experiments as well as the large scatter of data observed in the literature, we will analyze below different physical sources that can result in this phenomenon.

\section{A. The conduction band nonparabolicity effect}

The effect of the conduction band nonparabolicity in the triangular quantum well formed at the $\mathrm{AlGaN} / \mathrm{GaN}$ heterointerface can be evaluated according to the two-band approximation of Ando et al..$^{34}$

$$
m_{E}{ }^{*} / m_{0}{ }^{*}=\left[1+4\left(\left\langle E_{K}\right\rangle+E_{F}\right) / E_{g}\right]^{1 / 2},
$$

where $m_{0}{ }^{*}$ is the effective mass at the band edge, $E_{g}$ is the bandgap energy $\left[3.51 \mathrm{eV}\right.$ for $\mathrm{GaN}$ (Ref. 11)], $E_{F}$ $=\pi \hbar^{2} n_{S} / m_{E}{ }^{*}$ is the Fermi energy, and $\left\langle E_{K}\right\rangle$ is the average kinetic energy of the confined 2DEG. The $\left\langle E_{K}\right\rangle$ in the triangular well can be estimated as $E_{0} / 3$, and the quantization energy $E_{0}$ can be calculated from the simple Hartree approximation. ${ }^{35}$ Actually, all theoretical models use an idealized picture of the band structure. In reality, the depth and curvature of the quantum well in $\mathrm{AlGaN} / \mathrm{GaN}$ depend on several factors (including the charge polarization at the interface, alloy disorder in the barrier, residual impurities, and structural defects) and could be quite different even for samples with the same 2DEG density. Our estimations were performed for the investigated $\mathrm{AlGaN} / \mathrm{GaN}$ samples using Eq. (1) with $n_{s}=1 \times 10^{13} \mathrm{~cm}^{-2}$ and $m_{0}{ }^{*}=0.20 m_{0}$ give $m_{E}{ }^{*} / m_{0}{ }^{*} \approx 1.10$, i.e., only $10 \%$ effective mass enhancement caused by the conduction band nonparabolicity. Even using the more accurate Fang-Howard variational model ${ }^{34}$ for $\left\langle E_{K}\right\rangle$ the calculation does not considerably change the result.

\section{B. The polaron effect}

Taking into account the strong coupling between electrons and the longitudinal optical (LO) phonon in polar GaN with a large Fröhlich coupling constant $(\alpha=0.49),{ }^{5}$ one could expect the influence of the polaron on the effective mass of 2D electrons confined in the $\mathrm{AlGaN} / \mathrm{GaN}$ quantum well. However, the screening of the electron-LO-phonon interaction as well as the quantum confinement of the electron wave function strongly suppress the polaron effect. According to the Das Sarma result, ${ }^{36}$ the polaron contribution to the effective mass enhancement can be expressed as

$$
m_{P}{ }^{*}=m_{E}{ }^{*}[1+(\pi \alpha / 8) F(\gamma, \beta)],
$$

where parameters $\gamma=q_{\mathrm{TF}} / q_{0}$ and $\beta=b / q_{0}$ determine the strength of the screening and quantum confinement effects, respectively, $q_{0}=\left(2 m_{b}{ }^{*} \omega_{\mathrm{LO}} / \hbar^{2}\right)^{1 / 2}$ is the LO-phonon wave number $\left[\hbar \omega_{\mathrm{LO}}=90 \mathrm{meV}\right.$ (Ref. 11)], $q_{\mathrm{TF}}=m_{E}{ }^{*} e^{2} / \kappa \hbar^{2}$ is the Thomas-Fermi screening wave number, $e$ is the electron discharge, $\hbar$ is the reduced Plank constant, $\kappa$ is the dielectric constant, and $b$ is the variational parameter of Ando et $a l^{34}$ For $\mathrm{AlGaN} / \mathrm{GaN}$ samples under study, the factor $F(\gamma, \beta)$ $\approx 0.3$, and our estimations using Eq. (2) give $m_{P}{ }^{*} / m_{E}{ }^{*}$ $\approx 1.05$. This value is of the order of experimental error. Therefore, the contribution of the polaron effect is small and can be neglected.

\section{The effect of magnetic field}

The cyclotron-resonance and the $\mathrm{SdH}$ oscillations are the two experimental techniques most widely used for the determination of the 2DEG effective mass. In the cyclotronresonance experiment, the separation of the adjacent Landau levels, $\hbar \omega_{c}$, measured as a function of the magnetic field $B$, allows to estimate the effective mass $m_{E}{ }^{*}$ from the fit using the relation $m_{E}{ }^{*}=e B / \omega_{c}$. Due to the conduction band nonparabolicity, the spacing between Landau levels becomes irregular and, as a consequence, the measured effective mass manifests a dependence on the magnetic field. In the case of the $\mathrm{SdH}$ measurements, the situation is quite different. The $\mathrm{SdH}$ oscillations occur because the conductivity, $\sigma_{x x}$, and hence magnetoresistivity, $\rho_{x x}$, depend on the density of states at the Fermi energy, $g\left(E_{F}\right)$, both through the number of carriers and through the scattering rate. In 2DEG systems where only a single subband is occupied, the oscillatory part of the magnetoresistivity can be expressed as ${ }^{37}$

$$
\Delta \rho_{x x}=\rho_{0}\left[X_{T} / \sin \mathrm{h}\left(X_{T}\right)\right] \exp \left(-\pi / \omega_{c} \tau_{q}\right),
$$

where $\rho_{0}=m_{E}{ }^{*} / n_{S} e^{2} \tau_{\text {tr }}$ is the zero-field resistivity, $X_{T}$ $=2 \pi^{2} k_{B} T / \hbar \omega_{c}$ is the temperature-damping factor, $\tau_{\text {tr }}$ $=\mu m^{*} / e$ is the transport lifetime derived from the electron Hall mobility $\mu$, and $\tau_{q}$ is the quantum lifetime characterizing the disorder, which can be expressed in terms of the Dingle temperature $T_{D}=\hbar / 2 \pi k_{B} \tau_{q}$, where $k_{B}$ is the Boltzmann constant. Then, the effective mass $m_{E}{ }^{*}$ can be derived from the temperature dependence of the $\mathrm{SdH}$ oscillation amplitude at the fixed magnetic field using the simplified fitting function: ${ }^{34}$

$$
\ln (A / T)=C-2 \pi^{2} k_{B} T m_{E}{ }^{*} / e \hbar B,
$$

where $A$ is the amplitude of $\mathrm{SdH}$ oscillations and $C$ is the temperature independent constant. However, it should be noted that the approximation (4) is not necessarily valid in the whole temperature range where the $\mathrm{SdH}$ oscillations are observed.

The results of the fitting performed for sample B using Eqs. (3) and (4) of the measured data are shown in Fig. 3. It is clearly seen that the extrapolation according to Eq. (4) (dashed lines in Fig. 3) in the low-temperature limit brings the explicitly overestimated values $(0.22-0.25) m_{0}$ and reveals the dependence on the magnetic field, whereas Eq. (3) (solid curves in Fig. 3) gives the value $\sim 0.2 m_{0}$ for all three 


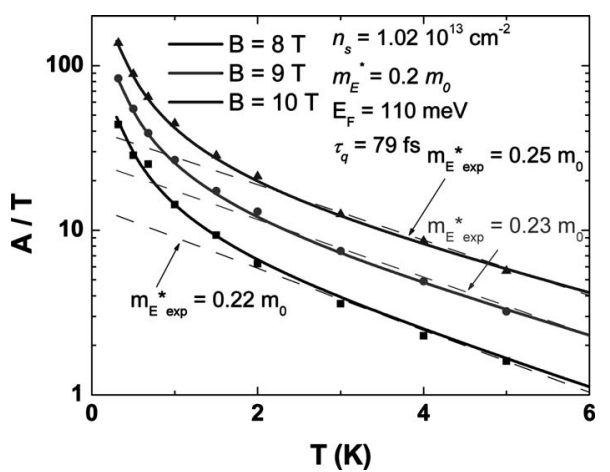

FIG. 3. Amplitudes of $\mathrm{SdH}$ oscillations in $\mathrm{AlGaN} / \mathrm{GaN}$ heterostructure (sample B) as a function of temperature for magnetic fields $B=8 \mathrm{~T}$ (squares), $9 \mathrm{~T}$ (circles), and $10 \mathrm{~T}$ (triangles). The solid and dashed lines are fits using Eqs. (3) and (4), respectively.

different magnetic fields. As already mentioned above, the peak of the SdH oscillations occurs when the sequential Landau level crosses the Fermi level. Thus, the amplitude of each $\mathrm{SdH}$ oscillation corresponds to the effective mass $m_{E}{ }^{*}$ at the Fermi energy, and there is no reason to expect any magnetic field dependence of the effective mass, at least for Landau levels with high indices.

\section{The effect of wave-function hybridization}

Because of the spatial extension, the tail of the wave function of 2D electrons occupying the lowest subband can penetrate into the layers confining the triangular quantum well at the $\mathrm{AlGaN} / \mathrm{GaN}$ interface. The electron wavefunction penetration into the barrier then causes the effective electron mass increase toward the barrier bulk mass value $\left[\sim 0.27 m_{0}\right.$ for $\mathrm{AlGaN}$ (Ref. 11)]. In other words, the penetration leads to the enhancement of the 2DEG effective mass.

To analyze this effect, we calculated the energy band structure, density of states, and the envelope wave functions of $2 \mathrm{D}$ electrons in the conduction band for our $\mathrm{AlGaN} / \mathrm{GaN}$ samples, using a self-consistent solution of the Schrödinger and Poisson equations. The model employs a tight-binding real-time Green's function approach based on the effective mass approximation and electron-electron interaction within a Hartree-self-consistent potential (for details, see Ref. 38).

The results of calculations are shown in Fig. 4. It confirms the experimental findings that only one level with the energy $E_{1}$ below the Fermi level $E_{F}$ is occupied. Due to the

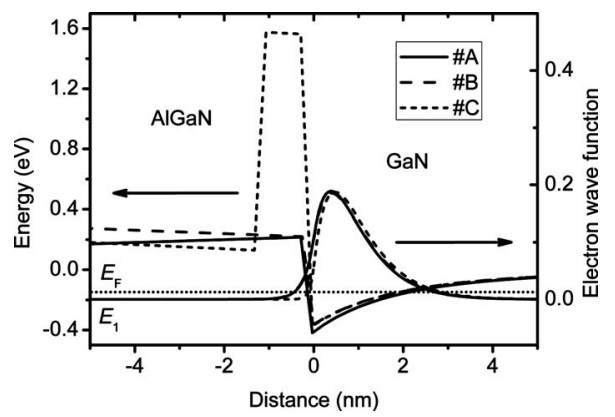

FIG. 4. Self-consistently calculated conduction band potential profile, Fermi level, and electron wave-function distribution for the investigated AlGaN/ $\mathrm{GaN}$ heterostructures (samples A-C). Interface is at $z=0$. large density of the 2DEG, the spatial redistribution of the confined electron $\langle z\rangle$ is a few nanometers. The most noticeable difference in the behavior of the electron wave function is observed for samples A and C. As is seen, the AlN interlayer between $\mathrm{GaN}$ and $\mathrm{AlGaN}$ in sample $\mathrm{C}$ reduces the wave-function penetration into the barrier for a fixed well width. On the opposite, in sample A with the single AlGaN/ $\mathrm{GaN}$ interface, the wave function extends deeper into the barrier due to the 2D electrons in the first subband, which are close to the interface, resulting in a larger effective mass as compared to sample $\mathrm{C}$. These results are in good agreement with the experimental data in Table I.

The effective mass of 2D electrons in the $i$ th subband of the conduction band, $m_{i}$, can be defined (Ref. 39, p.162) as

$$
\frac{1}{m_{i}}=\int d z \chi_{i}^{2}(z) \frac{1}{m(z)}
$$

where $\chi_{i}$ is the electron wave function of the $i$ th subband, $m(z)$ is the position-dependent effective mass of the 2DEG, and $z$ is the coordinate normal to the heterointerface $(x-y$ plane). In the case of the flatband the right part of the Eq. (5) can be solved analytically (Ref. 39, p.78) as

$$
\frac{1}{m_{i}}=\frac{1}{m_{w}}\left[1-P_{b}\left(E_{i}\right)\right]+\frac{1}{m_{b}} P_{b}\left(E_{i}\right),
$$

where $m_{w}$ is the effective mass in the quantum well, $m_{b}$ is the effective mass in the barrier layer, $P_{b}\left(E_{i}\right)$ is the probability of finding 2D electrons in the barrier at the level with energy $E_{i}$, and $m_{i}$ corresponds to value of experimentally obtained $m_{e}$.

We determined the probabilities of the 2DEG wavefunction penetration into the barrier by self-consistent calculation for our $\mathrm{AlGaN} / \mathrm{GaN}$ samples and for HEMT GaNbased heterostructures reported in the literature ${ }^{15-17,21-23,28,29}$ containing all necessary information for the detailed simulation.

To find the integral probability of the wave-function penetration into the barrier by the variational method, we used the trial wave functions in the following form: ${ }^{12}$

$$
\begin{aligned}
& \chi_{b}(z)=C_{1} \exp \left(k_{b} z\right), \quad z \leq 0, \\
& \chi_{w}(z)=C_{2}\left(z+z_{0}\right) \exp (-b z / 2), \quad z \geq 0,
\end{aligned}
$$

where $\chi_{b}$ is the electron wave function in the barrier; $\chi_{w}$ is the electron wave function in the quantum well; $C_{1}, C_{2}$, and $z_{0}$ are constants; $k_{b}=\sqrt{2 m_{b} V^{\prime}{ }_{b} / \hbar^{2}}$ is the parameter of the wave-function decay in the barrier; $V^{\prime}{ }_{b}$ is the effective conduction band offset at the heterojunction measured from the first electron level $E_{1} ; m_{b}$ and $m_{w}$ are the effective masses in the barrier and quantum well, respectively. For $m_{w}$ and $n_{S}$, we used the actual values of the effective mass and the sheet density of the 2DEG derived from experimental data. The variational parameter $b$ was determined using the relation of Ando et $a l .{ }^{34}$ as $b=\sqrt[3]{24 \pi m_{w} n_{S} e^{2} / \kappa \hbar^{2}}$.

Taking into account the boundary conditions requiring the continuity of the electron flux at the interface

$$
\chi_{b}(0)=\chi_{w}(0), \quad \chi_{b}^{\prime}(0)=\chi_{w}^{\prime}(0)
$$

and the normalization rule for the electron wave function: 
TABLE II. Calculated parameters of 2DEG in AlGaN/GaN heterostructures.

\begin{tabular}{lccc}
\hline \hline & \multicolumn{3}{c}{ Sample } \\
\cline { 2 - 4 } & $\mathrm{A}$ & $\mathrm{B}$ & $\mathrm{C}$ \\
\hline$m_{i}\left(m_{0}\right)$ & 0.220 & 0.200 & 0.190 \\
$m_{b}\left(m_{0}\right)$ & 0.266 & 0.260 & 0.400 \\
$E_{1}(\mathrm{meV})$ & 200 & 170 & 195 \\
$V_{b}(\mathrm{meV})$ & 635 & 582 & 1935 \\
$P_{b}(\%)$ & 3.58 & 3.45 & 0.398 \\
\hline \hline
\end{tabular}

$$
\int_{-\infty}^{0} d z\left|\chi_{b}\right|^{2}+\int_{0}^{+\infty} d z\left|\chi_{w}\right|^{2}=1
$$

we have determined values of constants $z_{0}, C_{2}$, and $C_{1}$ :

$$
\begin{aligned}
& z_{0}=1 /\left(k_{b}+b / 2\right), \quad C_{2}=\sqrt{1 /\left(z_{0}^{2} / 2 k_{b}+2 / b^{3}+2 z_{0} / b^{2}+z_{0}^{2} / b\right)}, \\
& \quad \text { and } C_{1}=z_{0} C_{2},
\end{aligned}
$$

necessary for the probability calculation of finding $2 \mathrm{D}$ electrons in the barrier and quantum well using Eqs. (7) and (8):

$$
\begin{gathered}
P_{b}\left(\chi_{b}\right)=\int_{-\infty}^{0} d z\left|\chi_{b}\right|^{2}, \quad P_{w}\left(\chi_{w}\right)=\int_{0}^{+\infty} d z\left|\chi_{w}\right|^{2}, \\
\text { and } P_{b}=\frac{P_{b}\left(\chi_{b}\right)}{P_{b}\left(\chi_{b}\right)+P_{w}\left(\chi_{w}\right)} .
\end{gathered}
$$

The results of probability calculations of the 2DEG wave-function penetration into the barrier for given AlGaN/ GaN samples are summarized in Table II and are used in Eq. (6) for the calculation of the effective mass. Evidently, they are in good agreement with experimental data of Table I. Really, the probability of finding $2 \mathrm{D}$ electrons in the barrier can be specifically small in the case of the AlN interlayer (sample C) but normally it increases with increasing 2DEG confinement in the single-heterojunction $\mathrm{AlGaN} / \mathrm{GaN}$ (samples A and B).

The summary of the data for the $2 \mathrm{DEG}$ effective mass in the investigated $\mathrm{AlGaN} / \mathrm{GaN}$ heterostructures is shown in Fig. 5. The points with the reference marks ${ }^{15-17,21-23,28,29}$ correspond to experimental data, taken from the literature and the points with the letter marks $\mathrm{A}, \mathrm{B}$, and $\mathrm{C}$ represent our experimental data. The solid line is the fit of the 2DEG effective mass dependence on the probability of wave-function

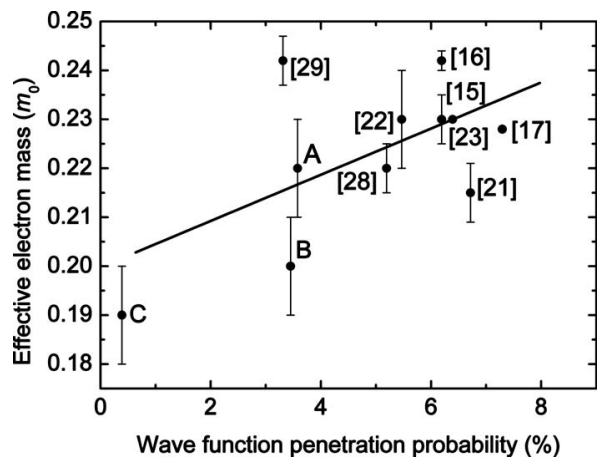

FIG. 5. 2DEG effective mass $m_{E}{ }^{*}$ as a function of the probability of the wave-function penetration into the barrier of $\mathrm{AlGaN} / \mathrm{GaN}$ heterostructures. penetration into the barrier of the studied $\mathrm{AlGaN} / \mathrm{GaN}$ heterostructures. The vertical bars correspond to the estimated measurement errors. Figure 5 shows that we reach a much more coherent picture for the 2DEG effective mass than we have in Fig. 1. For the totally nontransparent $\mathrm{AlGaN}$ barrier $\left(P_{b} \rightarrow 0\right)$ the value of the AlGaN/GaN 2DEG effective mass approaches the value for bulk GaN $\left(m^{*} \rightarrow 0.20 m_{0}\right)$. This result additionally supports the revealed finding that the origin of the 2DEG effective mass enhancement is the result of wave-function hybridization and proves the dominant role of the electron wave-function penetration in the $\mathrm{AlGaN}$ barrier layer. Thus, the hybridization of the wave function describes very well the effective mass enhancement in the case of each specific band structure in $\mathrm{AlGaN} / \mathrm{GaN}$ with different electron density and different spatial confinement.

\section{CONCLUSIONS}

In summary, we investigated the dependence of the inplane effective mass of the 2DEG formed in nominally undoped AlGaN/GaN HEMT-like structures at the AlGaN-GaN heterojunction as a function of the quantum confinement. The factors affecting the 2DEG effective mass enhancement, the sheet carrier density, conduction band nonparabolocity, polaron effect, magnetic field effect, and the electron wavefunction hybridization, were analyzed and evaluated. It is found that in most cases the conduction band nonparabolicity effect is overestimated, and the wave-function penetration into the AlGaN barrier should be taken into account. The hybridization of the wave function is determined to play the dominant role. The band edge effective mass of the AlGaN/ GaN 2DEG is deduced to be $(0.2 \pm 0.01) m_{0}$.

\section{ACKNOWLEDGMENTS}

This work was supported in part by the National Academy of Sciences of Ukraine, by the Ukrainian Ministry of Education and Science, by the Deutsche Forschungsgemeinshaft (Contract No. KL1342), and by the Office of Naval Research (Grant No. N00014-01-0828).

${ }^{1}$ P. Rinke, M. Winkelnkemper, A. Qteish, D. Bimberg, J. Neugebauer, and M. Scheffler, Phys. Rev. B 77, 075202 (2008).

${ }^{2}$ A. S. Barker, Jr. and M. Ilegems, Phys. Rev. B 7, 743 (1973).

${ }^{3}$ M. Suzuki, T. Uenoyama, and A. Yanase, Phys. Rev. B 52, 8132 (1995).

${ }^{4}$ P. Perlin, E. Litwin-Staszewska, B. Suchanek, W. Knap, J. Camassel, T. Suski, R. Piotrzkowski, I. Grzegory, S. Porowski, E. Kaminska, and J. C. Chervin, Appl. Phys. Lett. 68, 1114 (1996).

${ }^{5}$ M. Drechsler, D. M. Hofmann, B. K. Meier, T. Detchprohm, H. Amano, and I. Akasaki, Jpn. J. Appl. Phys., Part 2 34, L1178 (1995).

${ }^{6}$ W. J. Moore, J. A. Freitas, Jr., and R. J. Molnar, Phys. Rev. B 56, 12073 (1997).

${ }^{7}$ A. M. Witowski, K. Pakula, J. M. Baranowski, M. L. Sadowski, and P. Wyder, Appl. Phys. Lett. 75, 4154 (1999).

${ }^{8}$ S. Shokhovets, G. Gobsch, and O. Ambacher, Appl. Phys. Lett. 86, 161908 (2005).

${ }^{9}$ B. K. Meyer, D. Volm, A. Graber, H. C. Alt, T. Detchprohm, A. Amano, and I. Akasaki, Solid State Commun. 95, 597 (1995).

${ }^{10}$ D. Fritsch, H. Schmidt, and M. Grundmann, Phys. Rev. B 67, 235205 (2003).

${ }^{11}$ I. Vurgaftman and J. R. Meyer, J. Appl. Phys. 94, 3675 (2003).

${ }^{12}$ V. M. Mitin, V. A. Kochelap, and M. A. Strocio, Quantum Heterostructures: Microelectronics and Optoelectronics (Cambridge University Press, Cambridge, 1999).

${ }^{13}$ Y. J. Wang, R. Kaplan, H. K. Ng, K. Doverspike, D. K. Gaskill, T. Ikedo, 
I. Akasaki, and H. Amono, J. Appl. Phys. 79, 8007 (1996).

${ }^{14}$ W. Knap, H. Alause, J. M. Bluet, J. Camassel, J. Young, M. Asif Khan, Q. Chen, S. Huant, and M. Shur, Solid State Commun. 99, 195 (1996).

${ }^{15}$ W. Knap, S. Contreras, H. Alause, C. Skierbiszewski, J. Camassel, M. Dyakonov, J. Yang, Q. Chen, M. Asif Khan, F. H. Yang, M. Goiran, J. Leotin, and M. S. Shur, Appl. Phys. Lett. 70, 2123 (1997).

${ }^{16}$ W. Knap, E. Frayssinet, M. L. Sadowski, C. Skierbiszewski, D. Maude, V. Falko, M. Asif Khan, and M. S. Shur, Appl. Phys. Lett. 75, 3156 (1999)

${ }^{17}$ L. W. Wong, S. J. Cai, R. Li, K. Wang, H. W. Jiang, and M. Chen, Appl. Phys. Lett. 73, 1391 (1998).

${ }^{18}$ T. Y. Lin, H. M. Chen, M. S. Tsai, Y. F. Chen, F. F. Fang, C. F. Lin, and G C. Chi, Phys. Rev. B 58, 13793 (1998).

${ }^{19}$ S. Elhamri, R. S. Newrock, D. B. Mast, M. Ahoujja, W. C. Mitchel, J. M. Redwing, M. A. Tischler, and J. S. Flynn, Phys. Rev. B 57, 1374 (1998).

${ }^{20}$ T. Wang, J. Bai, S. Sakai, Y. Ohno, and H. Ohno, Appl. Phys. Lett. 76, 2737 (2000).

${ }^{21}$ A. Saxler, P. Debray, R. Perrin, S. Elhamri, W. C. Mitchel, C. R. Elsass, I. P. Smorchkova, B. Heying, E. Haus, P. Fini, J. P. Ibbetson, S. Keller, P. M. Petroff, S. P. DenBaars, U. K. Mishra, and J. S. Speck, J. Appl. Phys. 87, 369 (2000).

${ }^{22}$ A. F. Braña, C. Diaz-Paniagua, F. Batallan, J. A. Carrido, E. Muñoz, and F Omnes, J. Appl. Phys. 88, 932 (2000).

${ }^{23}$ D. R. Hang, C.-T. Liang, C. F. Huang, Y. H. Chang, Y. F. Chen, H. X Jiang, and J. Y. Lin, Appl. Phys. Lett. 79, 66 (2001).

${ }^{24}$ Z.-F. Li, W. Lu, S. C. Chen, S. Holland, C. M. Hu, D. Heitmann, B. Shen, Y. D. Zheng, T. Someya, and Y. Arakawa, Appl. Phys. Lett. 80, 431 (2002).

${ }^{25}$ S. Syed, M. J. Manfra, Y. J. Wang, H. L. Stormer, and R. J. Molnar, Phys. Rev. B 67, 241304(R) (2003).
${ }^{26}$ S. Syed, J. B. Heroux, Y. J. Wang, M. J. Manfra, R. J. Molnar, and H. L. Stormer, Appl. Phys. Lett. 83, 4553 (2003).

${ }^{27}$ W. Knap, V. I. Fal'ko, E. Frayssinet, P. Lorenzini, N. Grandjean, D. Maude, G. Karczewski, B. L. Brandt, J. Łusakowski, I. Grzegory, M. Leszczy'nski, P. Prystawko, C. Skierbiszewski, S. Porowski, X. Hu, G. Simin, M. Asif Khan, and M. S. Shur, J. Phys.: Condens. Matter 16, 3421 (2004).

${ }^{28}$ K. S. Cho, T.-Y. Huang, C.-P. Huang, Y.-H. Chiu, C.-T. Liang, Y. F. Chen, and I. Lo, J. Appl. Phys. 96, 7370 (2004).

${ }^{29}$ K. S. Cho, T.-Y. Huang, H.-S. Wang, M.-G. Lin, T.-M. Chen, C.-T. Liang, Y. F. Chen, and I. Lo, Appl. Phys. Lett. 86, 222102 (2005).

${ }^{30}$ N. Tang, B. Shen, M. J. Wang, Z. J. Yang, K. Xu, G. Y. Zhang, T. Lin, B. Zhu, W. Z. Zhou, and J. H. Chu, Appl. Phys. Lett. 88, 172115 (2006).

${ }^{31}$ Z. Bougrioua, I. Moerman, L. Nistor, B. Van Daele, E. Monroy, T. Palacios, F. Calle, and M. Leroux, Phys. Status Solidi A 195, 93 (2003).

${ }^{32}$ J. R. Shealy, V. Kaper, V. Tilak, T. Prunty, J. A. Smart, B. Green, L. F. Eastman, J. Phys.: Condens. Matter 14, 3499 (2002).

${ }^{33} \mathrm{~N}$. Nastase, H. Hardtdegen, R. Schmidt, H. Bay, H. Lüth, A. Alam, and M. Heuken, Phys. Status Solidi A 188, 647 (2001).

${ }^{34}$ T. Ando, A. B. Fowler, and F. Stern, Rev. Mod. Phys. 54, 437 (1982).

${ }^{35}$ M. A. Brummell, R. J. Nicholas, L. C. Brunel, S. Huant, M. Baj, J. C. Portal, M. Razeghi, M. A. Di Forte-Poisson, K. Y. Cheng, and A. Y. Cho, Surf. Sci. 142, 380 (1984).

${ }^{36}$ S. Das Sarma, Phys. Rev. B 27, 2590 (1983).

${ }^{37}$ P. T. Coleridge, R. Stoner, and R. Fletcher, Phys. Rev. B 39, 1120 (1989).

${ }^{38}$ K. M. Indlekofer, E. Dona, J. Malindretos, M. Bertelli, M. Kocan, A. Rizzi, and H. Lüth, Phys. Status Solidi B 234, 769 (2002).

${ }^{39}$ G. Bastard, Wave Mechanics Applied to Semiconductor Heterostructures (Les Editions de Physique, Paris, 1988). 